\title{
A Nomogram to Predict Prognostic Value of Red Cell Distribution Width in Patients with Esophageal Cancer
}

\author{
Gui-Ping Chen, ${ }^{1}$ Ying Huang, ${ }^{2}$ Xun Yang, ${ }^{3}$ and Ji-Feng Feng ${ }^{3}$ \\ ${ }^{1}$ Department of Oncological Surgery, Zhejiang Cancer Hospital, Hangzhou 310022, China \\ ${ }^{2}$ Department of Nursing, Zhejiang Cancer Hospital, Hangzhou 310022, China \\ ${ }^{3}$ Department of Thoracic Surgery, Zhejiang Cancer Hospital, Hangzhou 310022, China
}

Correspondence should be addressed to Ji-Feng Feng; fengjif@163.com

Received 23 December 2014; Accepted 31 March 2015

Academic Editor: Sydney C. W. Tang

Copyright (C) 2015 Gui-Ping Chen et al. This is an open access article distributed under the Creative Commons Attribution License, which permits unrestricted use, distribution, and reproduction in any medium, provided the original work is properly cited.

\begin{abstract}
Objectives. The prognostic value of inflammatory index in esophageal cancer (EC) was not established. In the present study, we initially used a nomogram to predict prognostic value of red cell distribution width (RDW) in patients with esophageal squamous cell carcinoma (ESCC). Methods. A total of 277 ESCC patients were included in this retrospective study. Kaplan-Meier method was used to calculate the cancer-specific survival (CSS). A nomogram was established to predict the prognosis for CSS. Results. The mean value of RDW was $14.5 \pm 2.3 \%$. The patients were then divided into two groups: RDW $\geq 14.5 \%$ and RDW $<14.5 \%$. Patients with RDW $<14.5 \%$ had a significantly better 5-year CSS than patients with RDW $\geq 14.5 \%$ (43.9\% versus $23.3 \%, P<0.001$ ). RDW was an independent prognostic factor in patients with ESCC $(P=0.036)$. A nomogram could be more accurate for CSS. Harrell's c-index for CSS prediction was 0.68. Conclusion. RDW was a potential prognostic biomarker in patients with ESCC. The nomogram based on CSS could be used as an accurately prognostic prediction for patients with ESCC.
\end{abstract}

\section{Introduction}

Esophageal cancer (EC) is the 8th most common cancer and the 4th leading cause of cancer death worldwide [1]. The incidences vary widely in different countries and regions [2]. In China, the most common pathological type is squamous cell carcinoma (SCC), in contrast to the predominance of adenocarcinoma (AC) in the West $[3,4]$. Therefore, a study that takes into account the predominance of esophageal squamous cell carcinoma (ESCC) in China is more and more important.

It is widely accepted that systemic inflammatory response (SIR) plays an important role in cancer progression $[5,6]$. Red cell distribution width (RDW) is a widely used laboratory parameter for inflammatory disease [7]. Recent studies revealed that RDW is associated with prognosis in several cancers, such as lung cancer [8] and prostate cancer [9]. However, to our knowledge, no studies regarding the prognostic value of RDW in EC are available.

Therefore, the aim of the current study was to investigate the prognostic role of RDW in patients with ESCC.
In addition, we attempt to establish a predictive nomogram to predict the survival prediction in patients with ESCC.

\section{Patients and Methods}

From July 2006 to December 2008, a retrospective analysis was conducted for patients with ESCC who underwent radical esophagectomy. The inclusion criteria were as follows: (1) ESCC confirmed by histopathology and classified by the 7th edition of TNM classification [10]; (2) curative esophagectomy (Ivor Lewis procedure or McKeown procedure) with standard lymphadenectomy (two-field or three-field lymphadenectomy) [11, 12]; (3) limited disease without distant metastasis; (4) without preoperative neoadjuvant therapy; (5) without previous anti-inflammatory medicines; and (6) preoperative laboratory results obtained before esophagectomy. At last, 277 patients were enrolled in the current study. Ethical approval was obtained from the Ethical Committees of Zhejiang Cancer Hospital (Hangzhou, China). 
TABLE 1: Comparison of baseline clinical characteristics based on RDW.

\begin{tabular}{|c|c|c|c|c|c|c|}
\hline & Cases $(n)$ & RDW (\%) & $P$ value & & & $P$ value \\
\hline & & $($ mean $\pm S D)$ & & $<14.5(n)$ & $\geq 14.5(n)$ & \\
\hline Age (years) & & & 0.759 & & & 0.931 \\
\hline$\leq 60$ & 158 & $14.5 \pm 2.3$ & & 107 & 51 & \\
\hline$>60$ & 119 & $14.4 \pm 2.3$ & & 80 & 39 & \\
\hline Gender & & & 0.979 & & & 0.993 \\
\hline Female & 37 & $14.5 \pm 2.4$ & & 25 & 12 & \\
\hline Male & 240 & $14.5 \pm 2.3$ & & 162 & 78 & \\
\hline Tumor length $(\mathrm{cm})$ & & & $<0.001$ & & & 0.003 \\
\hline$\leq 3.0$ & 78 & $13.6 \pm 1.7$ & & 63 & 15 & \\
\hline$>3.0$ & 199 & $14.8 \pm 2.4$ & & 124 & 75 & \\
\hline Tumor location & & & 0.901 & & & 0.906 \\
\hline Upper/middle & 143 & $14.5 \pm 2.3$ & & 97 & 46 & \\
\hline Lower & 134 & $14.5 \pm 2.3$ & & 90 & 44 & \\
\hline Vessel invasion & & & 0.732 & & & 0.895 \\
\hline Negative & 232 & $14.5 \pm 2.3$ & & 157 & 75 & \\
\hline Positive & 45 & $14.4 \pm 2.3$ & & 30 & 15 & \\
\hline Differentiation & & & 0.401 & & & 0.493 \\
\hline Well/moderate & 222 & $14.4 \pm 2.2$ & & 152 & 70 & \\
\hline Poor & 55 & $14.7 \pm 2.6$ & & 35 & 20 & \\
\hline T stage & & & $<0.001$ & & & $<0.001$ \\
\hline $\mathrm{T} 1-2$ & 97 & $13.6 \pm 2.1$ & & 79 & 18 & \\
\hline T3-4 & 180 & $14.9 \pm 2.3$ & & 108 & 72 & \\
\hline $\mathrm{N}$ stage & & & 0.056 & & & 0.012 \\
\hline No & 150 & $14.2 \pm 2.2$ & & 111 & 39 & \\
\hline N1-3 & 127 & $14.8 \pm 2.3$ & & 76 & 51 & \\
\hline
\end{tabular}

Patients were followed up with a clinical examination in our outpatient department every 3 to 6 months for the first 2 years after surgery and then annually. A cancer-specific survival (CSS) analysis was ascertained in the current study. The CSS was defined as the time from surgery to cancerrelated death. The median follow-up was 42.6 months.

2.1. Statistical Analysis. Statistical analysis was conducted with SPSS 17.0 (SPSS Inc., Chicago, IL, USA) and R 3.1.2 software (Institute for Statistics and Mathematics, Vienna, Austria). Independent $t$-tests and chi-squared tests were used to compare clinical characteristics and RDW. The CSS was calculated by the Kaplan-Meier method. Univariate and multivariable analyses were used to examine the prognostic factors. A receiver operating characteristic (ROC) curve was plotted to verify the accuracy of RDW for CSS prediction. The area under curve (AUC) was used as an estimation of diagnostic accuracy. A nomogram for possible prognostic factors (including age and sex) associated with CSS was established by $\mathrm{R}$ software, and the predictive accuracy was evaluated by Harrell's concordance index (c-index) $[13,14]$.
A $P$ value less than 0.05 was considered to be statistically significant.

\section{Results}

Among the 277 patients, 37 (13.4\%) were women and 240 $(86.6 \%)$ were men. The histogram of RDW is shown in Figure 1 . The mean value of RDW was $14.5 \pm 2.3 \%$. The upper normal range for RDW was $14.5 \%$. Then patients were divided into two groups: $\mathrm{RDW} \geq 14.5 \%$ and $\mathrm{RDW}<14.5 \%$. Clinicopathologic characters between the high and low groups for RDW were shown in Table 1.

Patients with RDW $<14.5 \%$ had a significantly better 5year CSS than patients with RDW $\geq 14.5 \%$ (43.9\% versus $23.3 \%, P<0.001$ ) (Figure 2). Then clinicopathological characters for CSS prediction were investigated by univariate and multivariate analyses. Our study demonstrated that RDW was an independent prognostic factor in patients with ESCC. $\mathrm{RDW} \geq 14.5 \%$ had a hazard ratio (HR) of $1.396(95 \%$ confidence interval (CI): 1.022-1.908, $P=0.036$ ) for CSS (Table 2). 


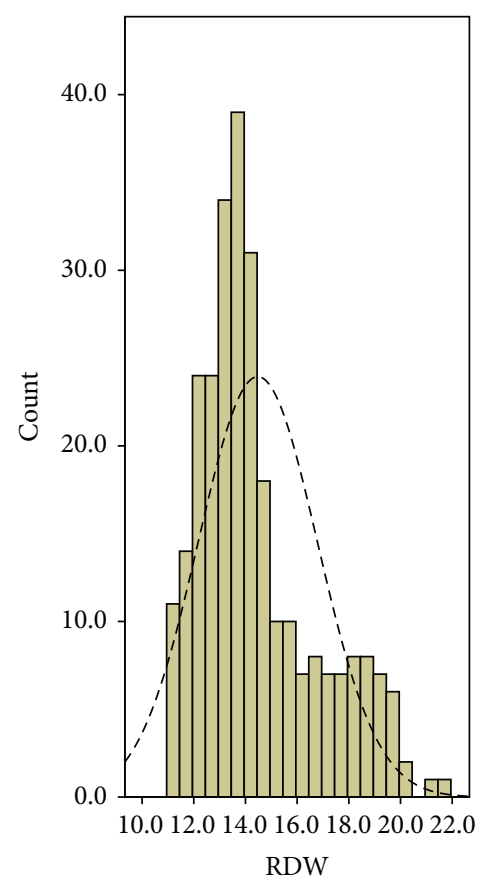

FIgURE 1: The histogram of the RDW.

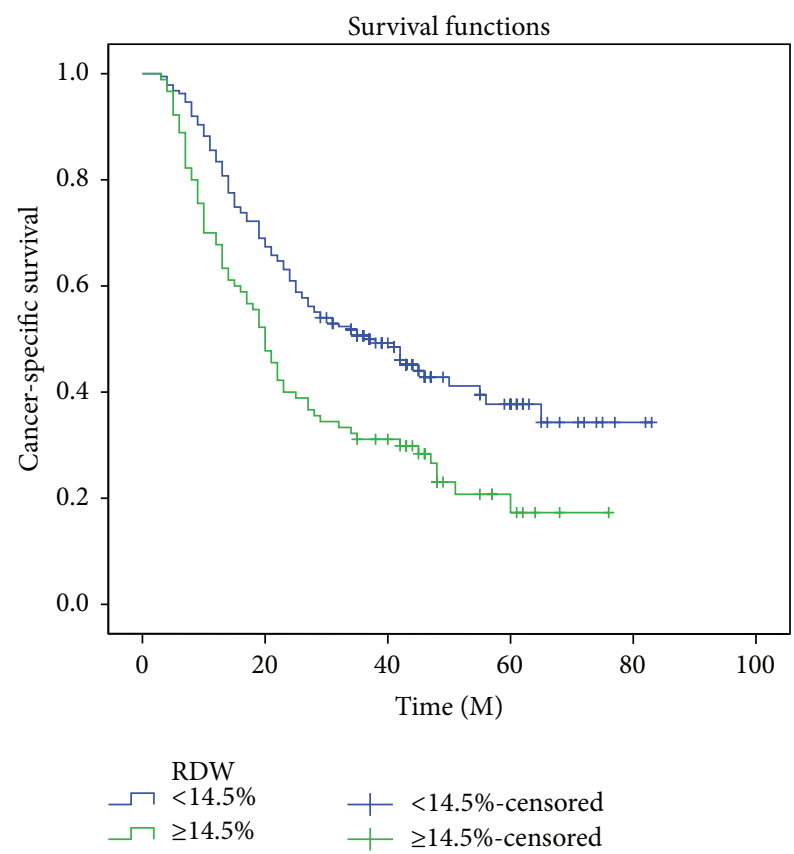

FIGURE 2: Kaplan-Meier CSS curves stratified by RDW. Patients with RDW $<14.5 \%$ had a significantly better 5 -year CSS than patients with RDW $\geq 14.5 \%$ ( $43.9 \%$ versus $23.3 \%, P<0.001$ ).

An ROC curve was also plotted to verify the accuracy of RDW for CSS prediction in the current study. The area under the curve (AUC) was 0.684 (95\% CI: $0.616-0.752, P<0.001$ ). It demonstrated that RDW (cut-off point: $14.5 \%$ ) predicts survival (CSS) with a sensitivity of $44.3 \%$ and a specificity of 77.7\% (Figure 3).

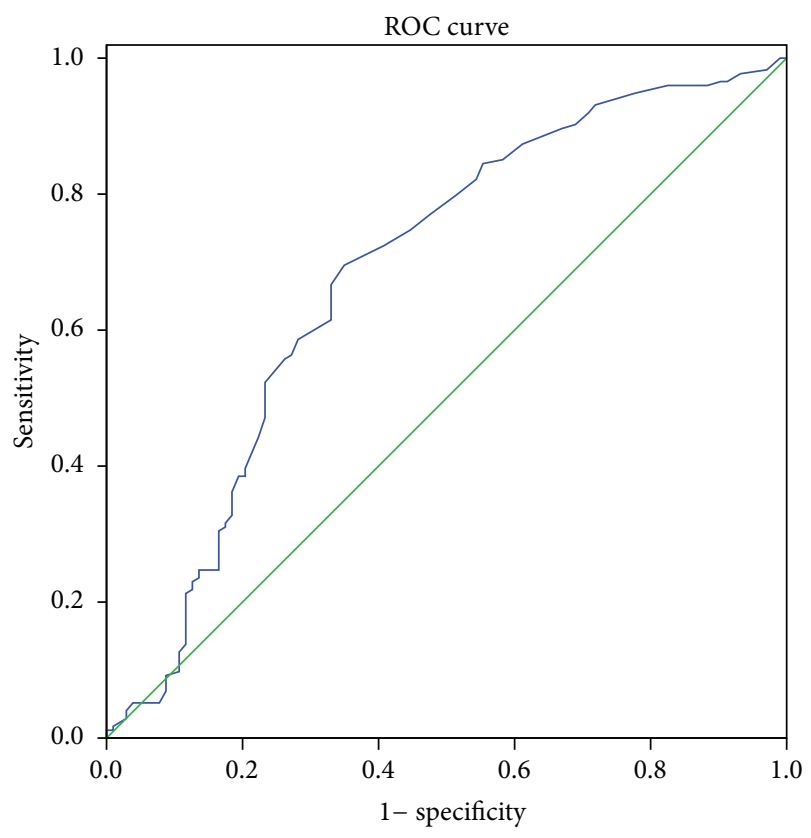

FIgURE 3: ROC curve for CSS prediction. A ROC curve plots the sensitivity on the $y$-axis against one minus the specificity on the $x$-axis. The area under curve (AUC) was used as an estimation of diagnostic accuracy. The AUC was 0.684 (95\% CI: 0.616-0.752, $P<0.001$ ). It demonstrated that RDW (cut-off point: 14.5\%) predicts survival with a sensitivity of $44.3 \%$ and a specificity of $77.7 \%$. Diagonal segments are produced by ties.

To predict the survival risk (CSS) for patients with ESCC, a novel model (nomogram) was established by prognostic factors combined with age and sex (Figure 4). It can predict the probability of death for patients with ESCC. Harrell's cindex for CSS prediction was 0.68 .

\section{Discussion}

To the best of our knowledge, this is the first study to determine the prognostic value of RDW in predicting prognosis for patients with ESCC. We concluded two major findings: (1) RDW was associated with cancer stage; (2) RDW was associated with cancer prognosis. In addition, our study was also the first attempt to establish a predictive nomogram to improve predictive accuracy based on RDW.

RDW is a parameter in complete blood cell count. It has been reported as an inflammatory biomarker $[7,15]$. The mechanism between inflammation and RDW has not been elucidated, but high levels of RDW are thought to be provoked by chronic inflammation and poor nutritional status $[16,17]$. Recent studies demonstrated that RDW is associated with prognosis in several cancers, such as lung cancer [8] and prostate cancer [9]. Therefore, in the current study, we investigated the prognostic role of RDW in patients with ESCC. We concluded that patients with RDW $<14.5 \%$ had a significantly better 5 -year CSS than patients with RDW $\geq 14.5 \%$ ( $43.9 \%$ versus $23.3 \%, P<0.001$ ). Because the values of RDW were positively associated with cancer stage (T stage 
TABLE 2: Univariate and multivariate analyses of CSS in ESCC patients.

\begin{tabular}{|c|c|c|c|c|c|c|}
\hline & CSS \% & $P$ value & $\begin{array}{c}\text { Univariate analysis } \\
\text { HR (95\% CI) }\end{array}$ & $P$ value & $\begin{array}{c}\text { Multivariate analysis } \\
\text { HR }(95 \% \text { CI })\end{array}$ & $P$ value \\
\hline Age (years) & & 0.579 & & 0.583 & - & - \\
\hline$\leq 60$ & 38.0 & & 1.000 & & & \\
\hline$>60$ & 36.1 & & $1.088(0.806-1.468)$ & & & \\
\hline Gender & & 0.182 & & 0.189 & - & - \\
\hline Female & 51.4 & & 1.000 & & & \\
\hline Male & 35.0 & & $1.387(0.851-2.260)$ & & & \\
\hline Tumor length $(\mathrm{cm})$ & & $<0.001$ & & 0.001 & & 0.385 \\
\hline$\leq 3.0$ & 53.8 & & 1.000 & & 1.000 & \\
\hline$>3.0$ & 30.7 & & $1.918(1.328-2.769)$ & & $1.198(0.797-1.799)$ & \\
\hline Tumor location & & 0.331 & & 0.337 & - & - \\
\hline Upper/middle & 41.3 & & 1.000 & & & \\
\hline Lower & 32.8 & & $1.157(0.859-1.558)$ & & & \\
\hline Vessel invasion & & 0.003 & & 0.003 & & 0.547 \\
\hline Negative & 40.5 & & 1.000 & & 1.000 & \\
\hline Positive & 20.0 & & $1.738(1.203-2.511)$ & & $1.125(0.767-1.652)$ & \\
\hline Differentiation & & 0.029 & & 0.033 & & 0.061 \\
\hline Well/moderate & 39.2 & & 1.000 & & 1.000 & \\
\hline Poor & 29.1 & & $1.476(1.033-2.109)$ & & $1.418(0.984-2.043)$ & \\
\hline T stage & & $<0.001$ & & $<0.001$ & & 0.011 \\
\hline $\mathrm{T} 1-2$ & 57.7 & & 1.000 & & 1.000 & \\
\hline T3-4 & 26.1 & & $2.371(1.668-3.369)$ & & $1.683(1.129-2.508)$ & \\
\hline $\mathrm{N}$ stage & & $<0.001$ & & $<0.001$ & & $<0.001$ \\
\hline No & 54.7 & & 1.000 & & 1.000 & \\
\hline N1-3 & 16.5 & & $2.846(2.089-3.877)$ & & $2.279(1.640-3.165)$ & \\
\hline Adjuvant therapy & & 0.121 & & 0.126 & - & - \\
\hline No & 40.3 & & 1.000 & & & \\
\hline Yes & 30.2 & & $1.277(0.934-1.746)$ & & & \\
\hline RDW (\%) & & $<0.001$ & & $<0.001$ & & 0.036 \\
\hline$<14.5$ & 43.9 & & 1.000 & & 1.000 & \\
\hline$\geq 14.5$ & 23.3 & & $1.719(1.268-2.331)$ & & $1.396(1.022-1.908)$ & \\
\hline
\end{tabular}

and $\mathrm{N}$ stage) in our study, it is not surprising that patients with higher RDW values had worse survival.

In the present study, we attempt to establish a predictive nomogram to predict the survival prediction based on RDW and other prognostic factors. We believe that our model could be a simple and easy tool for both the doctors and patients for estimating the survival in the absence of treatment in patients with ESCC. Thus, for example, a female ( 9 points) patient aged 60 years (38 points) with T2 (33 points), N1 (31 points), and $\mathrm{RDW} \geq 14.5 \%$ (33 points) would score 144 total points which converts to a risk probability for death of $57 \%$. Thus, we believe that nomogram based on CSS could be used as an accurately prognostic prediction for patients with ESCC.

Recent studies reported that SIR plays an important role in cancer progression $[5,6]$. However, the prognostic value of inflammatory index in EC was not established. Thus, more and more inflammatory indexes were confirmed. In our previous study, we showed that lymphopenia is an independent predictive factor in patients with ESCC [18]. However, we also demonstrated in limitations that diabetes mellitus, renal and/or hepatic failure, and other inflammatory diseases may potentially affect the lymphocytes. In the current study, therefore, we attempted to investigate the new inflammatory index (RDW) in patients with ESCC. In addition, we created a nomogram based on the RDW and other associated risk factors. This can be an edge over using lymphocyte count model. We believe that our model could be a simple and easy tool for both the doctors and patients for estimating the survival in the absence of treatment in patients with ESCC. Based on the findings, we recommend that RDW, a very common, easy, and simple marker, should be considered as a potential prognostic biomarker in patients with ESCC.

Several limitations should be acknowledged in the current study. First, the current study was a retrospective study 


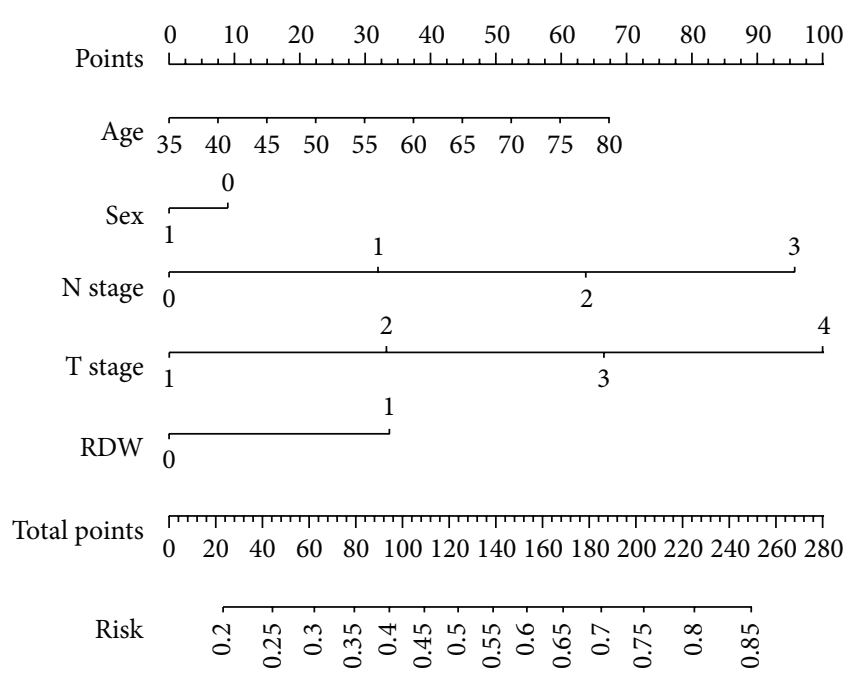

FIGURE 4: A nomogram predicts survival prediction based on RDW and other prognostic factors in patients with ESCC. The nomogram is used by totalling the points identified at the top of the scale for each independent factor. This total point score is then identified on the total points scale to determine the probability of risk prediction. Harrell's c-index for CSS prediction was 0.68 . For example, a female (9 points) patient aged 60 years (38 points) with T2 (33 points), $\mathrm{N} 1$ (31 points), and RDW $\geq 14.5 \%$ (33 points) would score 144 total points which converts to a risk probability for death of $57 \%$.

with a small sample. Second, the c-index showed that the model has a good accuracy but it is not perfect. Thus, there is still room for improvement of the predictive ability of the nomogram. The data in the current study was overlapped but from a different perspective. In our previous study, we mentioned the limitation that lymphopenia itself alone without other variables may not associate with the cancer prognosis. Thus, aim of current study was to investigate prognostic role of RDW. We concluded that RDW predicts survival similar to lymphocyte count and should be considered as an alternative to lymphocyte count. However, further studies are needed to illuminate the relationship between RDW and prognosis in patients with ESCC.

In summary, based on the findings of the current study, we concluded that RDW is a potential prognostic biomarkers in patients with ESCC. The nomogram based on CSS could be used as an accurately prognostic prediction for patients with ESCC.

\section{Conflict of Interests}

All authors declare no conflict of interests to disclose.

\section{References}

[1] R. Siegel, D. Naishadham, and A. Jemal, "Cancer statistics, 2013," CA: Cancer Journal for Clinicians, vol. 63, no. 1, pp. 11-30, 2013.

[2] J. Ferlay, H.-R. Shin, F. Bray, D. Forman, C. Mathers, and D. M. Parkin, "Estimates of worldwide burden of cancer in 2008: GLOBOCAN 2008," International Journal of Cancer, vol. 127, no. 12, pp. 2893-2917, 2010.
[3] F. Kamangar, G. M. Dores, and W. F. Anderson, "Patterns of cancer incidence, mortality, and prevalence across five continents: defining priorities to reduce cancer disparities in different geographic regions of the world," Journal of Clinical Oncology, vol. 24, no. 14, pp. 2137-2150, 2006.

[4] K. J. Napier, M. Scheerer, and S. Misra, "Esophageal cancer: a review pathogenesis, staging workup and treatment modalities," World Journal of Gastrointestinal Oncology, vol. 6, pp. 112-120, 2014.

[5] F. Balkwill and A. Mantovani, "Inflammation and cancer: back to Virchow?” The Lancet, vol. 357, no. 9255, pp. 539-545, 2001.

[6] A. Mantovani, P. Allavena, A. Sica, and F. Balkwill, "Cancerrelated inflammation," Nature, vol. 454, no. 7203, pp. 436-444, 2008.

[7] A. Yeşil, E. Şenateş, I. V. Bayoğlu, E. D. Erdem, R. Demirtunç, and A. O. K. Övünç, "Red cell distribution width: a novel marker of activity in inflammatory bowel disease," Gut and Liver, vol. 5, no. 4, pp. 460-467, 2011.

[8] Y. Koma, A. Onishi, H. Matsuoka et al., "Increased red blood cell distribution width associates with cancer stage and prognosis in patients with lung cancer," PLoS ONE, vol. 8, no. 11, Article ID e80240, 2013.

[9] S. Albayrak, K. Zengin, S. Tanik, H. Bakirtas, A. Imamoglu, and M. Gurdal, "Red cell distribution width as a predictor of prostate cancer progression," Asian Pacific Journal of Cancer Prevention, vol. 15, no. 18, pp. 7781-7784, 2014.

[10] T. W. Rice, V. W. Rusch, H. Ishwaran, and E. H. Blackstone, "Cancer of the esophagus and esophagogastric junction: datadriven staging for the seventh edition of the American Joint Committee on Cancer/International Union Against Cancer Cancer Staging Manuals," Cancer, vol. 116, no. 16, pp. 3763-3773, 2010.

[11] D. E. Low and A. Bodnar, "Update on clinical impact, documentation, and management of complications associated with esophagectomy," Thoracic Surgery Clinics, vol. 23, no. 4, pp. 535550, 2013.

[12] T. Ye, Y. Sun, Y. Zhang, and H. Chen, "Three-field or two-field resection for thoracic esophageal cancer: a meta-analysis," Annals of Thoracic Surgery, vol. 96, no. 6, pp. 1933-1941, 2013.

[13] M. W. Kattan, "Validating a prognostic model," Cancer, vol. 107, no. 11, pp. 2523-2524, 2006.

[14] F. E. Harrell, Regression Modeling Strategies, Springer, New York, NY, USA, 2001.

[15] Y. Beyazit, M. Kekilli, M. Ibis et al., "Can red cell distribution width help to discriminate benign from malignant biliary obstruction? A retrospective single center analysis," HepatoGastroenterology, vol. 59, no. 117, pp. 1469-1473, 2012.

[16] K. V. Patel, L. Ferrucci, W. B. Ershler, D. L. Longo, and J. M. Gurainik, "Red blood cell distribution width and the risk of death in middle-aged and older adults," Archives of Internal Medicine, vol. 169, no. 5, pp. 515-523, 2009.

[17] L. Ferrucci, J. M. Guralnik, R. C. Woodman et al., "Proinflammatory state and circulating erythropoietin in persons with and without anemia," American Journal of Medicine, vol. 118, no. 11, pp. 1288.e11-1288.e19, 2005.

[18] J. F. Feng, J. S. Liu, and Y. Huang, "Lymphopenia predicts poor prognosis in patients with esophageal squamous cell carcinoma," Medicine, vol. 93, no. 27, article e257, 2014. 


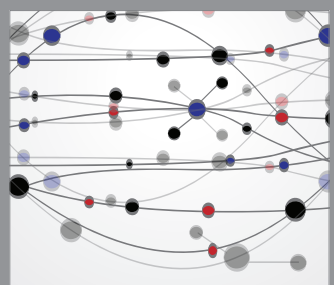

The Scientific World Journal
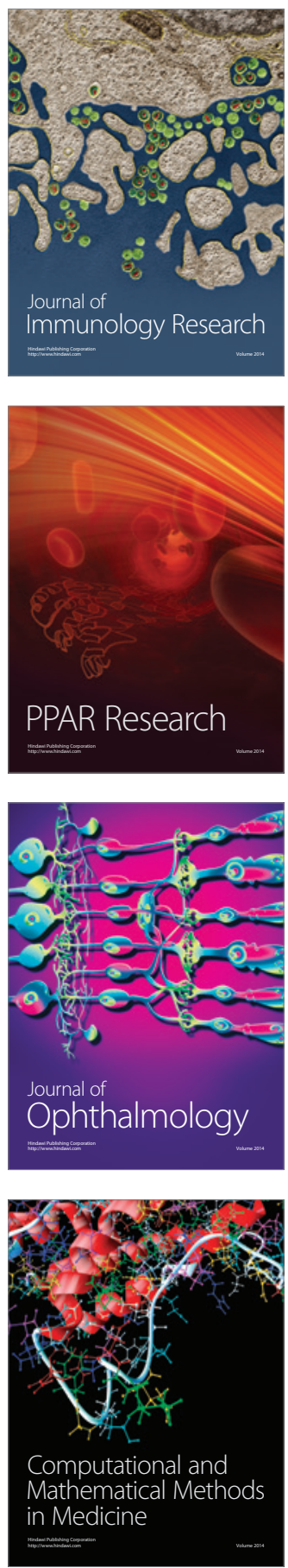

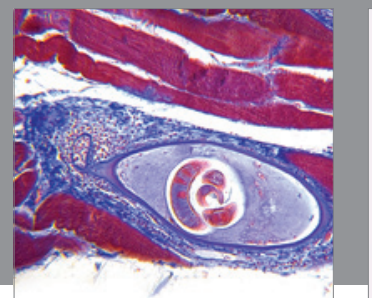

Gastroenterology

Research and Practice
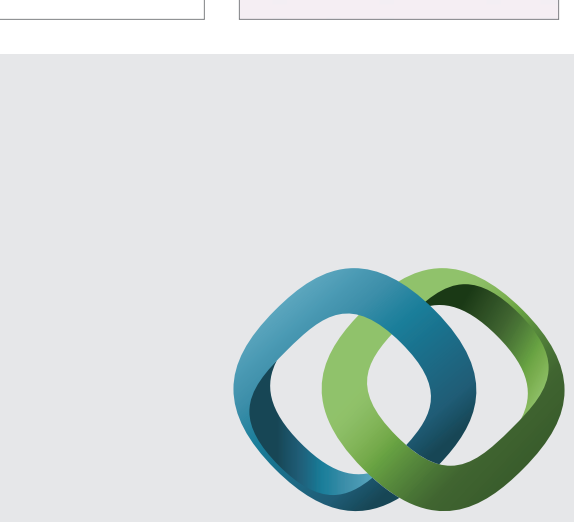

\section{Hindawi}

Submit your manuscripts at

http://www.hindawi.com
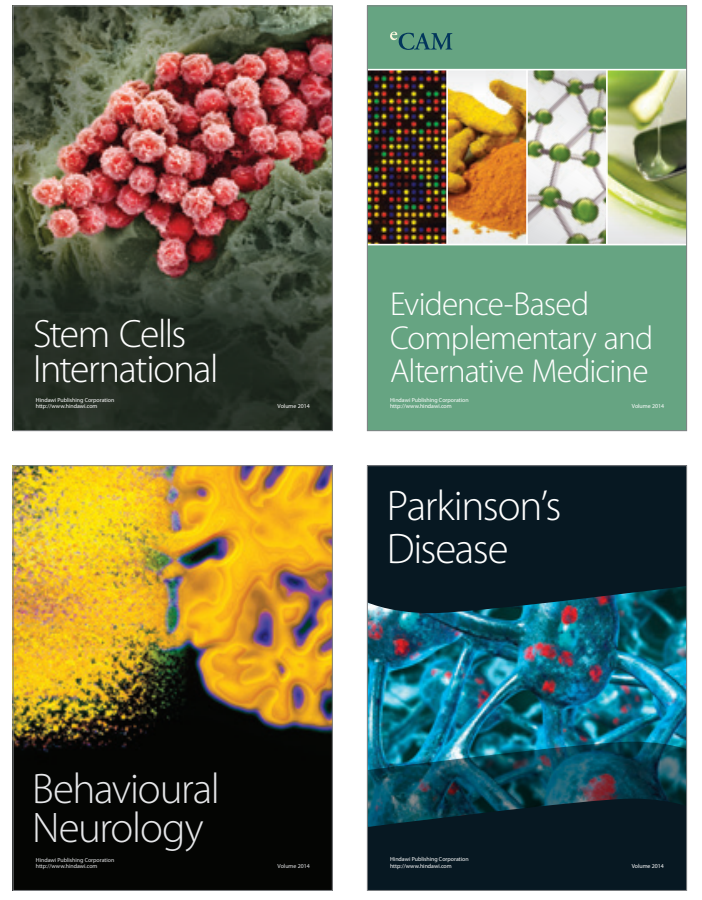
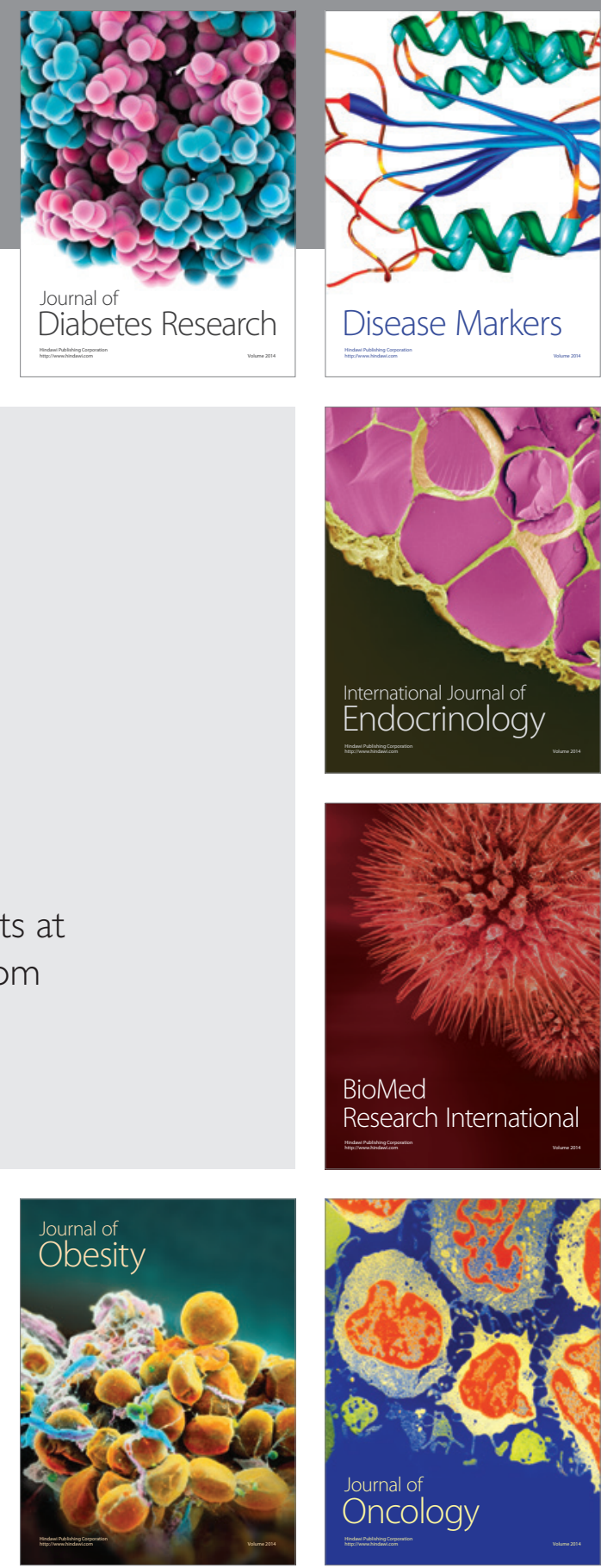

Disease Markers
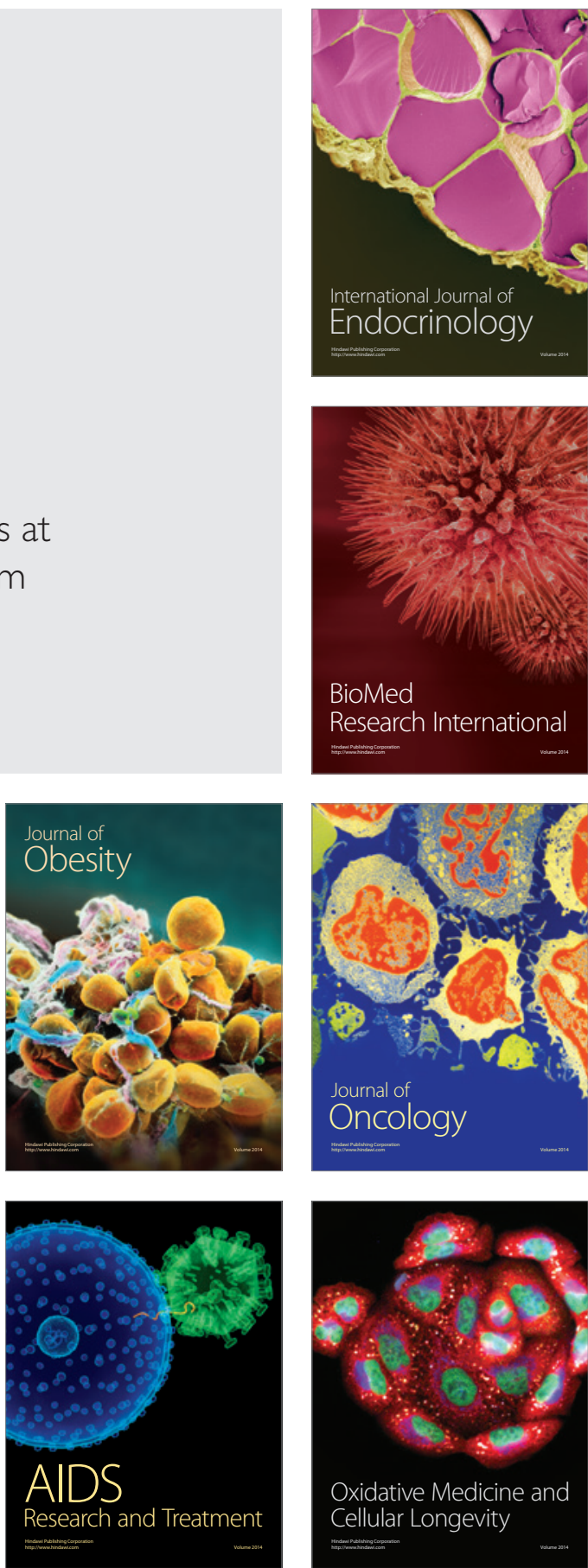